\title{
TRATAMENTO CIRÚRGICO DA HIPERTENSÃO ARTERIAL SECUNDÁRIA COM ORIGEM NA GLÂNDULA SUPRA-RENAL
}

\author{
SURGICAL TREATMENT OF SECONDARY ARTERIAL HYPERTENSION ORIGINATED \\ FROM ADRENAL GLAND
}

Ruy Garcia Marques, TCBC-RJ'1 ; Antonio Felipe Sanjuliani² $^{2}$

\section{INTRODUÇÃO}

Dentre as principais causas de hipertensão arterial secundária, despontam as afecções tumorais que acometem a glândula supra-renal, sejam localizadas no córtex aldosteronismo primário (síndrome de Conn) e síndrome de Cushing - ou na medula glandular - feocromocitoma. Embora, em sua maioria, esses tumores sejam benignos, a ressecção cirúrgica, curativa em cerca de $80 \%$ a $90 \%$ dos casos ${ }^{1,2}$, é necessária para eliminar os distúrbios humorais deles decorrentes e para impedir o crescimento da massa. Neste artigo, algumas particularidades acerca do tratamento cirúrgico dessas enfermidades serão focalizadas, enfatizando o preparo pré-operatório dos pacientes e a forma de abordagem atualmente preconizada.

\section{ADRENALECTOMIA}

A adrenalectomia pode ser realizada por dois métodos de acesso: o aberto (ou convencional) e o laparoscópico, ambos permitindo a abordagem transabdominal ou retroperitoneal das lesões.

A ressecção cirúrgica da glândula supra-renal pode ser total ou parcial. Quando o tumor é unilateral, geralmente se realiza a adrenalectomia total, mas, naqueles casos de ocorrência bilateral, deve-se sempre que possível almejar a preservação de parte do tecido glandular (adrenalectomia parcial), em ao menos um dos lados, para que se evite a insuficiência supra-renal pós-operatória e o indispensável uso de corticosteróides, por toda a vida.

No método aberto, as duas vias de acesso transabdominais mais utilizadas são a incisão mediana, supra- e/ou infra-umbilical, e a incisão subcostal, uni- ou bilateral, ambas permitindo a exploração da cavidade abdominal e possibilitando a ressecção de lesões bilaterais ou múltiplas. Especificamente para feocromocitomas, a incisão mediana permitiria, adicionalmente, a ressecção de eventuais paragangliomas. Essas incisões, pelo amplo campo operatório que propiciam, facilitam o controle vascular da glândula, minimizando a ocorrência de complicações operatórias. Entretanto, carreiam com si todo o potencial de morbidade inerente a qualquer operação que envolva a manipulação do conteúdo intra-abdominal. Em grandes tumores, muitos deles malignos, pode ser necessária a exploração cirúrgica através de incisões tóraco-abdominais, aumentando, sobremaneira, a morbidade associada ao procedimento.

As vias de acesso por lombotomias (nos flancos, por incisões póstero-laterais) ou por incisões posteriores (com o paciente posicionado em decúbito ventral), possibilitam a abordagem retroperitoneal das pequenas lesões da glândula supra-renal. A grande vantagem dessa abordagem é a diminuição da ocorrência de aderências e da lesão a órgãos intraabdominais. Por não envolverem a abertura da cavidade abdominal, habitualmente permitem recuperação rápida do paciente, com alta hospitalar mais precoce. Como grande inconveniente, contudo, constata-se a total impossibilidade da exploração da cavidade abdominal por essas incisões, possibilitando, eventualmente, que seja deixada uma outra tumoração, inicialmente insuspeita, determinando a perpetuação do quadro clínico. ${ }^{3}$ Quando utilizadas para tratamento de lesões bilaterais, após o término da operação em um dos lados, o paciente é reposicionado, de modo a se conduzir a operação contralateral. As incisões posteriores podem ser longitudinais ou oblíquas, com ou sem ressecção da $12 .{ }^{a}$ costela, mas, por oferecerem campo operatório restrito, seu uso vem declinando, mormente após a introdução da adrenalectomia laparoscópica.

Desde a sua primeira descrição, em $1992,{ }^{4}$ a adrenalectomia por vídeolaparoscópia tem tido utilização progressivamente maior, para tratamento de quase, todos os tumores da glândula supra-renal. Inicialmente, existiam apenas relatos isolados e seletivos de ressecções vídeolaparoscópicas para lesões tumorais da glândula suprarenal. À medida que o método foi se difundindo, com a disponibilização de novos instrumentos e a constatação de sua segurança, a operação minimamente invasiva foi ganhando adeptos. Ademais, propicia recuperação pós-operatória e retorno às atividades habituais mais precocemente, além da

1. Professor Adjunto Doutor e Chefe do Departamento de Cirurgia Geral da Faculdade de Ciências Médicas - UERJ; Coordenador da Disciplina de Técnica Operatória e Cirurgia Experimental - FCM - UERJ.

2. Professor Adjunto Doutor do Departamento de Medicina Interna e do Curso de Pós-Graduação em Fisiopatologia Clínica e Experimental CLINEX - Faculdade de Ciências Médicas - UERJ.

Recebido em 03/07/06

Aceito para publicação em 18/08/06

Conflito de interesses: nenhum

Fonte de financiamento: nenhuma

Trabalho realizado no Hospital Universitário Pedro Ernesto -Universidade do Estado do Rio de Janeiro. 
menor amplitude das incisões, provendo resultado cosmético superior, em comparação ao método aberto ou convencional. Este fato vem sendo atestado em grande número de estudos comparativos retrospectivos na literatura médica ${ }^{4-7}$.

A adrenalectomia vídeolaparoscópica pode ser realizada tanto por via transabdominal (com posicionamento do paciente em decúbito dorsal ou lateral, direito ou esquerdo) quanto retroperitoneal (com posicionamento do paciente em decúbito lateral direito ou esquerdo, de acordo com a lateralidade da lesão). A Tabela 1 mostra as vantagens, desvantagens e principais indicações das principais vias laparoscópicas para acesso às glândulas supra-renais.

Em recente revisão da literatura existente sobre o assunto (2004), Assalia \& Gagner(2004) analisando 2.550 procedimentos operatórios laparoscópicos sobre a glândula adrenal, relatados por diversos autores, entre 1990 e 2003, verificaram que a sua principal indicação é o aldosteronoma (síndrome de Conn), com 36,2\% dos casos, seguida pela síndrome de Cushing (19,1\%), por adenomas não-funcionantes $(18,2 \%)$ e pelo feocromocitoma $(18,0 \%){ }^{7}$

Nessa revisão, o percentual de complicações dos procedimentos laparoscópicos foi de 9,5\% (40\% de sangramento intra-e pós-operatório, 13\% de complicações infecciosas, incluindo infecção de ferida operatória, e 6,4\% de lesões em órgãos intra-abdominais, dentre outras), com taxa de conversão da operação para a via aberta de 3,6\%, notadamente devido ao sangramento intra-operatório incontrolável. O maior número de complicações, especialmente as lesões em órgãos intra-abdominais, bem como o emprego de maior tempo operatório, ocorreram mais freqüentemente no início das diversas séries revistas. Como ocorre em todas as situações em que se adota qualquer nova modalidade técnica, a denominada "curva de aprendizado" do método associa-se a um índice maior de complicações e a um período de tempo mais elevado para sua realização. Contudo, na atualidade, o índice potencial de com- plicações, bem como o tempo operatório, para ambos os métodos, é similar. Mesmo sob o aspecto do custoefetividade, a adrenalectomia vídeolaparoscópica, computando-se todos os seus benefícios, não parece diferir da operação por via aberta, havendo relatos de que essa relação pode ser até melhor, em favor da primeira opção ${ }^{8}$. Dentre as inúmeras casuísticas compiladas e analisadas por Assalia \& Gagner (2004), ${ }^{7}$ algumas relataram comparações de parâmetros selecionados acerca de adrenalectomias convencionais (abertas) vs. vídeolaparoscópicas, que estão sumarizados na Tabela 2.

Algumas contra-indicações relativas para a ressecção vídeolaparoscópica incluem risco cardiopulmonar muito elevado e coagulopatia de grande magnitude, intratável. Entretanto, na atualidade, as únicas contra-indicações absolutas são a presença de grandes tumores malignos, com nítida invasão para estruturas vizinhas, e feocromocitomas metastáticos para linfonodos periaórticos ${ }^{7,9}$. À exceção desses casos, todos os tumores da glândula supra-renal menores que $6 \mathrm{~cm}$ podem ser habilmente ressecados por vídeolaparoscópia (para alguns autores, tumores até $10-12 \mathrm{~cm}$ também são potencialmente ressecáveis) ${ }^{10-12}$. Para tumores maiores ou não totalmente determinados pelos métodos de imagem e quando há concomitância de tumores extra-adrenais, o método aberto convencional ainda permanece sendo a escolha inicial. Entretanto, mesmo em alguns desses casos, tem-se indicado a adrenalectomia vídeo-assistida, quando não se determina totalmente, no pré-operatório, se o tumor pode ser completamente ressecado por vídeolaparoscopia. Nesses casos, a dissecção é iniciada por laparoscopia, com conversão planejada para um procedimento aberto para completar a dissecção, ou com a realização de uma incisão abdominal para introdução da mão do cirurgião para auxiliar na dissecção e para retirada de tumoração com maior dimensão. ${ }^{3}$

Cabe ressaltar a realização de adrenalectomias laparoscópicas em regime ambulatorial, em pacientes com bai-

Tabela 1 - Comparação de vias de acesso laparoscópico às glândulas supra-renais.

\begin{tabular}{llll}
\hline Via de acesso & Vantagens & Desvantagens & Indicações \\
\hline
\end{tabular}

Transabdominal

Anterior e subcostal

\section{Retroperitoneal}

Lateral e posterior
Exposição direta e precoce da glândula, com dissecção do tumor sob completa visualização; a ligadura e secção da veia adrenal (principalmente à esquerda) é factível precocemente.
Esquerda: mobilização do

Ausência de dissecção e lesão de órgãos intra-abdominais; não propicia o surgimento de aderências; recuperação mais precoce. baço, cauda pancreática e flexura colônica esquerda. Direita: mobilização do fígado e da flexura colônica direita; o pólo superior da glândula é de difícil dissecção quando o tumor se estende para o fígado.

Tumores de tamanho variado, com ou sem extensão para o fígado (à direita) ou pâncreas (à esquerda); tumores bilaterais.

Notadamente por via posterior, pequeno espaço de trabalho; dificuldade para identificação da glândula e para acesso à veia adrenal. 
Tabela 2 - Adrenalectomia vídeolaparoscópica vs. convencional (modificado de Assalia \& Gagner). ${ }^{7}$

\begin{tabular}{|c|c|c|c|c|c|c|c|c|c|c|c|c|}
\hline \multirow[t]{2}{*}{ Casuística } & \multicolumn{2}{|c|}{$\begin{array}{c}\text { Número } \\
\text { de pacientes }\end{array}$} & \multicolumn{2}{|c|}{$\begin{array}{l}\text { Tamanhodo } \\
\text { tumor }(\mathbf{c m})^{*}\end{array}$} & \multicolumn{2}{|c|}{$\begin{array}{c}\text { Tempo } \\
\text { operatório }(\min )^{*}\end{array}$} & \multicolumn{2}{|c|}{$\begin{array}{c}\text { Perda } \\
\text { sangüínea (ml)* }\end{array}$} & \multicolumn{2}{|c|}{$\begin{array}{c}\text { Complicações } \\
(\%)\end{array}$} & \multicolumn{2}{|c|}{$\begin{array}{c}\text { Tempo de } \\
\text { internação (dias) }\end{array}$} \\
\hline & $\mathbf{A L}$ & $\mathbf{A C}$ & $\mathbf{A L}$ & $\mathrm{AC}$ & $\mathbf{A L}$ & $\mathrm{AC}$ & $\mathbf{A L}$ & $\mathrm{AC}$ & $\mathbf{A L}$ & $\mathbf{A C}$ & $\mathbf{A L}$ & $\mathrm{AC}$ \\
\hline 1 & 38 & 36 & 2,3 & 2,6 & 225 & 122 & 138 & 188 & 11 & 8 & 8,5 & 12,9 \\
\hline 2 & 42 & 38 & n.d. & n.d. & n.d. & n.d. & n.d. & n.d. & 0 & 11 & n.d. & n.d. \\
\hline 3 & 19 & 48 & 3,3 & 5,2 & 198 & 228 & n.d. & n.d. & 3 & 60 & 1,5 & 6,3 \\
\hline 4 & 22 & 17 & n.d. & n.d. & 288 & 270 & n.d. & n.d. & n.d. & n.d. & 1,7 & 6,7 \\
\hline 5 & 20 & 20 & $13,9 \dagger$ & $11,5 \dagger$ & 193 & 178 & 245 & 283 & 10 & 25 & 3,1 & 7,2 \\
\hline 6 & 21 & 20 & 3,2 & 9,2 & 206 & 177 & n.d. & n.d. & n.d. & n.d. & 2,2 & 6,1 \\
\hline 7 & 18 & 147 & 4,0 & 7,0 & 116 & 132 & n.d. & n.d. & 0 & 12 & 2,2 & 6,3 \\
\hline 8 & 12 & 7 & 2,0 & 6,4 & 116 & 166 & 132 & 278 & 8 & 0 & 2,1 & 5,4 \\
\hline 9 & 12 & 56 & 1,8 & 1,6 & 160 & 120 & 50 & 150 & 0 & 9 & 3,0 & 5,0 \\
\hline 10 & 21 & 17 & 1,8 & 2,5 & 219 & 140 & 183 & 266 & 29 & 76 & 2,7 & 6,2 \\
\hline 11 & 110 & 100 & $29,0 \ddagger$ & $28,6 \ddagger$ & 189 & 219 & 125 & 563 & 15 & 32 & 1,9 & 7,6 \\
\hline 12 & 24 & 28 & 3,6 & 2,9 & 188 & 139 & n.d. & n.d. & 16 & 39 & 4,0 & 7,5 \\
\hline 13 & 10 & 10 & n.d. & n.d. & 110 & 123 & n.d. & n.d. & 10 & 30 & 3,7 & 5,8 \\
\hline 14 & 19 & 19 & 2,5 & 3,3 & 164 & 151 & 109 & 263 & 5 & 38 & 2,3 & 5,1 \\
\hline 15 & 17 & 12 & n.d. & n.d. & 289 & 201 & 198 & 500 & 21 & 56 & 3,0 & 7,9 \\
\hline 16 & 36 & 23 & n.d. & n.d. & 158 & 85 & n.d. & n.d. & 6 & 52 & 3,5 & 8,5 \\
\hline 17 & 24 & 42 & 2,7 & 2,9 & 183 & 139 & 104 & 387 & 17 & 64 & 3,2 & 7,5 \\
\hline 18 & 50 & 50 & 2,9 & 2,9 & 167 & 127 & n.d. & n.d. & 6 & 72 & 3,1 & 5,7 \\
\hline 19 & 40 & 40 & 2,8 & 2,7 & 147 & 79 & 40 & 172 & 5 & 50 & 12,0 & 18,0 \\
\hline 20 & 28 & 25 & 1,7 & 3,7 & 375 & 123 & 370 & 345 & 25 & 8 & 12,0 & 18,2 \\
\hline Total & 581 & 753 & & & & & & & & & & \\
\hline Média & & & 2,8 & 4,1 & 184 & 162 & 154 & 309 & 10,9 & 35,8 & $2,9 \S$ & $7,2 \S$ \\
\hline
\end{tabular}

$A L$-adrenalectomia vídeolaparoscópica; $A C$-adrenalectomia convencional; $n$.d. - dados não disponíveis;

* valores médios; + volume em $\mathrm{cm}^{3} ;$ † peso em gramas; § excluidos os dados das casuísticas 1, 19 e 20, que apresentaram tempos excessivamente altos de internação hospitalar.

xo risco e com pequenos tumores (principalmente, aldosteronomas $)^{13,14}$. e, mesmo, com operações robóticas, ${ }^{15,16}$ com resultados satisfatórios.

O perfeito conhecimento da anatomia das glândulas supra-renais é indispensável para o tratamento das afecções cirúrgicas que nela se instalam. São glândulas pequenas, com cerca de 3 a 6 g, cada uma, apresentando duas porções claramente distintas: córtex e medula. $\mathrm{O}$ córtex apresenta coloração amarelo-brilhante, e é dividido em três camadas: glomerulosa, fasciculada e reticular. Na região glomerulosa, responsável pela produção de mineralocorticóides, notadamente aldosterona, é que se situam os aldosteronomas (aldosteronismo primário - síndrome de Conn); na região fasciculada, onde ocorre a produção de glicocorticóides, é que se localizam os tumores causadores da síndrome de Cushing (ACTH-independente). A medula glandular, local de feocromocitoma, contém células cromafíns, de tonalidade vermelho-escuro, secretoras de catecolaminas (adrenalina e noradrenalina). A glândula supra-renal direita, de formato piramidal, relaciona-se anteriormente com o fígado e com a veia cava inferior, e, posteriormente, com o músculo diafragma; a glândula esquerda, de formato semilunar, relaciona-se anteriormente com o pâncreas, baço e estômago, e, posteriormente, com o músculo diafragma. Apesar do rim direito situar-se mais inferiormente que o esquerdo, a glândula supra-renal direita localiza-se mais superiormente no retroperitônio, em relação à glândula contralateral ${ }^{17,18}$.

Em ambos os lados, sua irrigação arterial provém de ramos das artérias frênica inferior, aorta e renal, que originam as artérias supra-renais superior, média e inferior, respectivamente ${ }^{17,18}$. Em geral, essas artérias não oferecem maior dificuldade para controle operatório. Contudo, por serem de pequeno calibre e muito friáveis, podem ser inadvertidamente lesadas durante qualquer procedimento cirúrgico sobre as glândulas.

A drenagem venosa se dá por uma única veia, mas que se dirige para locais distintos, conforme a lateralidade glandular. A veia supra-renal esquerda é tributária da veia renal ipsilateral, e se entende anteriormente sobre a glândula, sendo de fácil visualização durante a manipulação do órgão. Entretanto, à direita, além de mais curta, a veia supra-renal desemboca na parede póstero-lateral da veia cava inferior, o que a torna de mais difícil acesso ${ }^{17,18}$. Antes de sua manipulação, recomenda-se controle vascular proximal e distal da veia cava inferior, o que minimiza os riscos para a sua dissecção, ligadura e secção.

Os vasos linfáticos, mais abundantes na medula glandular, geralmente acompanham as veias supra-renais, dirigindo-se aos linfonodos que margeiam os grandes vasos abdominais $^{17,18}$. 


\section{FEOCROMOCITOMA}

De todas as causas de hipertensão arterial secundária, a fisiopatologia do feocromocitoma é a melhor estudada, e o seu diagnóstico é, freqüentemente, o mais prontamente reconhecido. Esses tumores, compostos por células cromafíns, são capazes de secretar, armazenar e liberar catecolaminas na corrente sangüínea, fazendo parte das chamadas células APUD (amine precursor uptake and decarboxilation), de origem neuroectodérmica. Estas células se localizam mais habitualmente na medula supra-renal, nos plexos neurais simpáticos paragangliomas (plexos celíaco, mesentérico, renal, adrenal, hipogástrico, testicular e para-vertebral) e no órgão de Zuckerkandl (coleção de células para-aórticas, situadas junto à bifurcação da aorta) mas também podem ser encontradas na região cervical torácica ${ }^{19}$.

O feocromocitoma é um tumor neuroendócrino potencialmente letal, mais prevalente em mulheres de faixa etária intermediária, entre 30-50 anos de idade, freqüentemente como lesão solitária e benigna, mais comumente à direita que à esquerda, numa proporção de 5:3. ${ }^{20}$ Conhecido como o "tumor dos 10\%", 10\% dessas lesões podem ser bilaterais, múltiplas e extra-adrenais, $10 \%$ surgem em crianças, $10 \%$ são familiares, $10 \%$ são malignas e $10 \%$ apresentam recorrência no pós-operatório. ${ }^{3,21-23}$ Embora geralmente benignos, 2\% a 10\% dos tumores localizados nas glândulas adrenais e $20 \%$ a $40 \%$ dos extra-adrenais são malignos, ressaltando-se que, em crianças, é maior a possibilidade de ocorrência de malignidade. A caracterização de malignidade, entretanto, só pode ser comprovada pela presença de metástases, haja vista que, mesmo nos tumores benignos, pode ocorrer invasão local. ${ }^{3,23}$

Charles Mayo e Cesar Roux, em 1926, foram os primeiros a relatar a ressecção de feocromocitomas ${ }^{24}$. Desde essa época, o tratamento desses tumores persiste como um grande desafio para os cirurgiões, devido à importância do quadro clínico desencadeado e à necessidade estrita de cuidados pré e per-operatórios extremamente específicos. Até a cerca de 50 anos, a mortalidade operatória, nesses casos, aproximava-se dos $30 \%$, mas com a crescente percepção de suas complicações potenciais e com o uso de extenso armamentário farmacológico, esse percentual, atualmente, é inferior a $2 \%{ }^{25}$.

A seleção de pacientes para procedimentos abertos ou laparoscópicos deve ser individualizada. A adrenalectomia por via aberta continua sendo a preferida para pacientes com grandes tumores, naqueles casos em que haja suspeita de malignidade, em massas irregulares, em tumorações extraadrenais ou múltiplas, e quando há ausência de plano de clivagem com órgãos vizinhos ou envolvimento nodal periaórtico, fundamentado em exames de imagem, como tomografia computadorizada, ressonância magnética ou cintilografia com metaiodobenzilguanidina. Para tumores menores do que $6 \mathrm{~cm}$, uni- ou bilaterais, a ressecção por laparoscopia tem se tornado, progressivamente, a opção de escolha. Com a prática, o tempo operatório passa a ser similar, para ambas as vias de acesso, mas com uma importante redução do tempo de internação hospitalar em pacientes submetidos ao acesso laparoscópico ${ }^{3,5}$.
A primeira adrenalectomia por vídeolaparoscópica para feocromocitoma foi realizada em $1992 .{ }^{4}$ O maior obstáculo à sua pronta generalização deveu-se ao receio de que o pneumoperitônio por $\mathrm{CO}_{2}$, parte indispensável do procedimento vídeo- laparoscópico, pudesse desencadear crises hipertensivas com ameaça à vida dos pacientes. Entretanto, com a sua cada vez mais contumaz utilização, tem-se verificado que, com uma perfeita integração per-operatória de cirurgiões e anestesistas, não existe maior risco para essa ocorrência, com diversos autores mostrando que a ressecção vídoelaparoscópica associa-se a alterações hemodinâmicas similares, ${ }^{26}$ ou até menores, ${ }^{27}$ em comparação ao método de acesso convencional.

\section{Preparação do paciente para a operação}

A ressecção de um feocromocitoma ou de um paraganglioma bioquimicamente ativo apresenta grande potencial para complicações per- e pós-operatórias, haja vista a liberação de catecolaminas durante a manipulação do tumor. Efeitos hemodinâmicos e metabólicos significantes estão associados com um súbito decréscimo dos níveis de catecolaminas após a ressecção do tumor ${ }^{28}$. Avanços nas técnicas de localização dos tumores, preparação e manuseio anestésico adequados resultaram em evolução cirúrgica e pós-operatória significante, com diminuição notável da morbimortalidade operatória, notadamente nos últimos 30 $\operatorname{anos}^{19}$

O preparo do paciente com feocromocitoma inclui a instituição de bloqueio alfa-adrenérgico, e, em algumas situações, bloqueio beta-adrenérgico. A utilização de antagonistas-alfa parece ser responsável por boa parte do decréscimo da mortalidade perioperatória ${ }^{23}$. A fenoxibenzamina (dibenzyline $^{\circledR}$ ), um antagonista alfa-adrenérgico de longa ação, tem sido considerado o fármaco de escolha para controlar as alterações da pressão sangüínea e seus sintomas associados. Ela bloqueia os receptores alfa-adrenérgicos de forma nãocompetitiva e dificulta a ação das catecolaminas liberadas pelo tumor, além de permitir expansão de volume intravascular, mas, para tal, necessita ser utilizada por duas ou três semanas ${ }^{23}$. É iniciada antes da operação, ainda em regime ambulatorial. A dose inicial é de $10 \mathrm{mg}$, por via oral, duas vezes ao dia, combinada com monitorização não-invasiva da pressão arterial nas posições supina e sentada. A dose é aumentada, por incrementos de 10 mg diários, até que ocorra estabilização da pressão arterial e redução nos sintomas, com dose média de 40 mg/ dia (até $100 \mathrm{mg} / \mathrm{dia}$ ), com a maioria dos pacientes requerendo 10-14 dias de tratamento, antes da operação ${ }^{19,25}$. Contudo, não existe evidência de que o tratamento pré-operatório com fenoxibenzamina por mais do que 14 dias seja mais eficaz do que por quatro a sete dias, ao menos no que se refere ao efeito na pressão arterial per-operatória, na freqüência de pulso e na ocorrência de arritmias cardíacas ${ }^{28}$.

Elevações significantes da pressão arterial ainda podem ocorrer no per-operatório, especialmente durante a manipulação do tumor, a despeito de adequado bloqueio-alfa préoperatório $^{23,29}$. Podem ocorrer hipotensão ortostática e taquicardia reflexa, o último secundário à inibição dos receptores alfa-2 pré-sinápticos em neurônios pós-gangliônicos, 
resultando em liberação de noradrenalina ${ }^{23}$. O bloqueio alfaadrenérgico também pode contribuir para o estado hipotensivo que se segue à remoção do tumor e pode mascarar a queda na pressão sangüínea, utilizada pelo cirurgião como parâmetro da completa remoção do tumor ${ }^{23}$.

Os efeitos da fenoxibenzamina dissipam-se em aproximadamente 36 horas (apresenta meia-vida $>24$ horas) e a administração de grande volume de fluidos intravenosos é freqüentemente necessária no período pós-operatório. Os pacientes tornam-se mais sonolentos nas primeiras 24 horas após a operação, o que pode se dever a bloqueio alfa-2adrenérgico central persistente ${ }^{30}$.

Na ausência de arritmias, o pré-tratamento com agentes bloqueadores beta-adrenérgicos não é rotineiramente necessário, embora a associação de bloqueio alfa e beta possa permitir a recuperação do intenso estresse cardíaco e da cardiomiopatia induzidos por catecolaminas, que ocorre em até $60 \%$ desses pacientes ${ }^{22,31}$. Quando indicado, o bloqueio beta-adrenérgico é iniciado após a constatação clínica de eficácia do bloqueio-alfa, em pacientes que apresentam taquicardia persistente ou taquicardia reflexa relacionada ao início do bloqueio alfa, e naqueles com arritmias cardíacas. Entretanto, a eficácia do bloqueio beta-adrenérgico na prevenção de arritmias per-operatórias tem sido questionada ${ }^{32}$. É importante iniciar o bloqueio alfa antes do bloqueio betaadrenérgico, para evitar a situação de agonismo-beta, em resposta ao bloqueio-beta, em que o paciente apresenta intensa vasoconstriç̧ão do excesso alfa-adrenérgico, com risco de ocorrência de hipertensão extrema e aumento da sobrecarga cardíaca, que pode ocasionar edema pulmonar, eventualmente agravado pelo efeito inotrópico positivo do bloqueiobeta $^{23,33}$.

O labetalol, um bloqueador alfa- e beta-adrenérgico, tem sido utilizado, na dose de até $200 \mathrm{mg}$, por via oral, quatro vezes ao dia, mas, ocasionalmente, pode precipitar o surgimento de crises hipertensivas. Outra opção é o uso de propranolol, com ação beta-adrenérgica específica, na dose de $40-320 \mathrm{mg} / \mathrm{dia}^{23,25}$.

Antagonistas alfa-1-adrenérgicos competitivos, como prazosin (6-20 $\mathrm{mg} / \mathrm{dia})$, doxazosin e terazosin, também podem ser utilizados e parecem oferecer inúmeras vantagens potenciais, comparados à fenoxibenzamina (não disponível em nosso meio). Essa classe de medicamentos não produz taquicardia reflexa, apresenta menor duração de ação e pode ser rapidamente ajustada após a operação, decrescendo a duração da hipotensão pós-operatória ${ }^{23,30}$. Com o seu uso, vislumbra-se não ser necessária a utilização do bloqueio beta-adrenérgico, a menos que o paciente apresente um tumor secretante seletivo de adrenalina, haja vista que os receptores alfa-2adrenérgicos (que regulam a liberação de noradrenalina nas terminações nervosas cardíacas) não são antagonizados ${ }^{23,29}$. $\mathrm{O}$ uso combinado de fenoxibenzamina e prazosin também é utilizado, na tentativa de propiciar o controle da pressão arterial antes e durante a operação, e diminuir as complicações inerentes à hipotensão decorrente do uso isolado da fenoxibenzamina ${ }^{25}$.

Outra possibilidade seria o uso de fentolamina $\left(\right.$ Regitina $\left.^{\circledR}\right)$, por via endovenosa, ou mesmo por via oral
$\left(\operatorname{Vasomax}^{\circledR}\right)$, por sua rápida ação no controle da pressão arterial. Todavia, como seu efeito é pouco duradouro, não é rotineiramente utilizada no pré-operatório. Em contraposição, no per-operatório, durante a manipulação tumoral, esse efeito é altamente desejável e a fentolamina $(0,1 \mathrm{ml} / \mathrm{kg})$ constitui uma das opções preferenciais para diminuir a pressão arterial decorrente da liberação de catecolamina ${ }^{25}$.

Antagonistas do canal de cálcio podem ser utilizados com segurança em pacientes normotensos que, ocasionalmente, apresentem episódios de hipertensão paroxística. Esses medicamentos não produzem hipotensão ortostática, além de poderem prevenir a ocorrência de vasoespasmo coronariano e miocardite, induzidos pela liberação de catecolaminas ${ }^{19,23}$.

O tratamento com metirosina (alfa-metil-paratirosina) no pré-operatório resulta na depleção dos estoques de catecolamina do tumor, por inibição competitiva da tirosinahidroxilase, provendo diminuição da labilidade pressórica e da perda sangüínea intra-operatória ${ }^{1}$. Com a depleção de catecolaminas, diminui a possibilidade de que o tumor reaja à estimulação ${ }^{1}$. Esse fármaco pode ser útil em pacientes com insuficiência cardíaca congestiva, em que o bloqueio alfaadrenérgico produz taquicardia ou o bloqueio beta-adrenérgico diminui a performance cardíaca. Sua associação com um agente bloqueador alfa-adrenérgico pode resultar em melhor controle pressórico e menor necessidade de uso de outras medicações anti-hipertensivas durante a operação, comparado ao bloqueio-alfa isolado ${ }^{34}$. A metirosina também pode ser empregada para alívio de sintomas, em pacientes portadores de tumores irressecáveis, em que o bloqueio com outros fármacos é insuficiente.

Em pacientes com feocromocitoma, o volume intravascular encontra-se diminuído. Isso se manifesta por hemoconcentração e por mudanças ortotásticas da pressão arterial. A vasoconstricção mediada por alfa-adrenérgicos e, possivelmente, a alteração da permeabilidade capilar, são tidas como responsáveis por esses achados ${ }^{25}$. O bloqueio alfaadrenérgico, por si, pode possibilitar ao paciente a restauração do volume intravascular. Se essa restauração é atingida, espera-se que a hemoconcentração resolva ou diminua antes da operação. Por vezes, contudo, há que se aumentar a oferta hídrica, tanto por via oral quanto por via intravenosa, por dois a três dias no pré-operatório.

Comumente, nos dias que antecedem a operação, se utiliza a sedação pré-operatória, com benzodiazepínicos ou outros agentes ansiolíticos, por diminuírem a ansiedade e prevenirem flutuações hemodinâmicas marcantes no período préoperatório imediato ${ }^{19}$.

Há que se mencionar que o bloqueio alfa-adrenérgico antes da ressecção do feocromocitoma, apesar de comumente realizado e geralmente recomendado ${ }^{35}$ não é universalmente aceito como absolutamente necessário para o sucesso perioperatório ${ }^{34,36}$. Boutros et a ${ }^{36}$ e Steinsapir et al ${ }^{34}$ acreditam que o avanço nas técnicas anestésicas e de monitorização, e a disponibilidade de fármacos de ação rápida na correção de alterações hemodinâmicas súbitas, tenham eliminado a necessidade do uso de bloqueio alfa-adrenérgico no preparo para ressecção desses tumores. Entretanto, a maioria dos autores 
continua preconizando a sua utilização, e, à luz do conhecimento atual, parece prudente se continuar utilizando o bloqueio alfa-adrenérgico, seguido pelo bloqueio betaadrenérgico, quando necessário, na preparação pré-operatória desses pacientes.

\section{Manuseio per-operatório}

O perfeito entrosamento entre a equipe cirúrgica e o anestesiologista é crucial para o sucesso do manuseio perioperatório de pacientes com feocromocitomas. A discussão pré-operatória de complicações potenciais e a habilidade para trabalhar como uma equipe, suprimem muitos dos problemas que podem surgir durante a operação.

Algumas considerações anestésicas são mandatórias e devem ser instituídas: (1) administração de ansiolíticos; (2) estabelecimento de um cateter para mensuração da pressão arterial, antes da indução; (3) estabelecimento de um cateter intravenoso para administração de medicamentos antihipertensivos; (4) mensuração contínua da pressão venosa central; (5) tratamento das flutuações hemodinâmicas com antihipertensivos e antagonistas beta-adrenérgicos; e (6) monitorização contínua para hipotensão e hipoglicemia, após $\mathrm{o}$ isolamento do tumor ${ }^{19}$.

Diversas técnicas anestésicas têm sido usadas para operações em pacientes portadores de feocromocitomas, mas sua descrição desvia-se da pretensão deste artigo. Infusões anti-hipertensivas de curta ação são preferidas para controle da pressão sangüínea, face à labilidade hemodinâmica antes da ressecção do tumor e a freqüência da hipotensão após.

No per-operatório, o tumor deve sofrer o mínimo possível de manipulação, para não desencadear liberação desenfreada de catecolaminas, com grande risco para o paciente, haja vista que pode ser de difícil controle. Sempre que o cirurgião for manusear o tumor, deve, primeiramente, avisar ao anestesiologista, para que ele se prepare para essa liberação de catecolaminas e possa agir, mesmo profilaticamente, para evitar riscos maiores para o paciente, sendo freqüentemente necessária a suplementação de bloqueadores alfa- e betaadrenérgicos ${ }^{30}$. Dripping de fentolamina (Regitina ${ }^{\circledR}$ ) ou nitroprussiato de sódio são comumente utilizados, durante esse tempo operatório.

Uma vez confirmada a localização do tumor adrenal, o primeiro passo é a identificação, dissecção, ligadura e secção da veia supra-renal, para impedir a liberação de catecolaminas para a circulação sangüínea. Tal tarefa é muito mais facilitada quando se trata de um tumor adrenal à esquerda, haja vista a situação anterior da veia adrenal, desde a porção central da glândula até sua desembocadura na veia renal ipsilateral. Em contraposição, quando o tumor se situa na glândula suprarenal direita, torna-se muito mais difícil a ligadura da veia, devendo-se tomar cuidados adicionais que assegurem pronto controle proximal e distal da veia cava inferior.

Com a ligadura da veia adrenal, freqüentemente ocorre intensa hipotensão, que deve ser coibida por extensa administração de fluidos. Por vezes, essa hipotensão é tão acentuada que enseja a necessidade de administração de agentes hipertensores, até mesmo de adrenalina, por via intravenosa. Procede-se, então, à ligadura dos ramos arteriais, liberação de possíveis aderências a órgãos vizinhos e ressecção da lesão. Alguns autores preconizam o uso de fenoldopam, no lugar da fentolamina ou do nitroprussiato, por sua vantagem em não apresentar metabolitos tóxicos. Esse medicamento age por estimulação dos receptores dopaminérgicos-1 que causam vasodilatação periférica enquanto, simultaneamente, propiciam o aumento do fluxo sangüíneo renal. Sua dose varia entre 0,2 e $0,8 \mu \mathrm{g} / \mathrm{kg} / \mathrm{min}^{3}$.

O uso intra-operatório de sulfato de magnésio, com administração em bolus de 40-60 mg/kg, antes da intubação traqueal, seguido por uma infusão de $2 \mathrm{~g} / \mathrm{h}$, tem sido advogada, pela inibição da liberação de catecolamina da medula adrenal e por suas propriedades anti-arrítmicas e vasodilatadoras ${ }^{37}$.

Com cuidadoso manuseio pré- e per-operatório, a incidência de complicações perioperatórias significantes não costuma ser de grande magnitude. Entretanto, a despeito de cuidadosa preparação para a operação, cerca de $30 \%$ dos pacientes ainda apresentam um curso perioperatório extremamente lábil. Pacientes com pressões sistólicas mais elevadas, com tumores de grande dimensão, com tumores recorrentes, altas concentrações urinárias de metanefrina e tempo operatório prolongado parecem ser mais propensos a apresentarem essas complicações ou evoluir para morte no período perioperatório ${ }^{2,29}$. Não há, entretanto, correlação dos níveis de catecolaminas com o risco dessa ocorrência ${ }^{29}$.

A recorrência pode ocorrer em cerca de $10 \%$ dos casos, e mesmo quando a lesão é benigna, a transformação maligna deve sempre ser considerada. Evidências clínica, bioquímica e radiográfica de recorrência podem levar anos para tornarem-se evidentes ${ }^{3}$.

Nos últimos 30 anos (1975-2005), 38 pacientes portadores de feocromocitomas foram operados no Hospital Universitário Pedro Ernesto (Departamento de Cirurgia Geral Faculdade de Ciências Médicas - UERJ). Há que se ressaltar que a maior parte dos pacientes com hipertensão arterial secundária com etiologia na glândula supra-renal, notadamente nos primeiros 20 anos dessa casuística, foi operada pelo Professor Humberto da Silva Peixoto, grande estudioso e entusiasta da Cirurgia Endócrina. Dos 38 tumores, 36 localizavam-se na glândula supra-renal (18 à direita, 14 à esquerda e quatro bilaterais), um em órgão de Zuckerkandl e outro em bexiga (Figura 1). Desses pacientes com tumorações localizadas em ambas as glândulas supra-renais, três (irmãs) apresentavam neoplasia endócrina múltipla tipo IIA (NEM-IIA), com concomitância de carcinoma medular da tireóide.
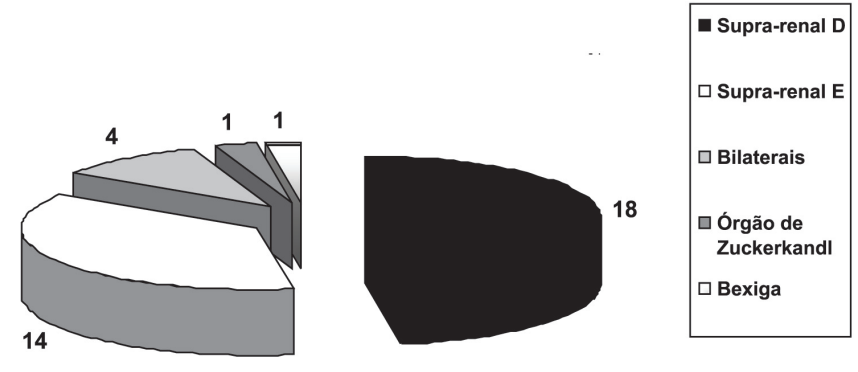

Figura 1 - Distribuição dos feocromocitomas (HUPE - UERJ). 
Tabela 3 - Distribuição dos pacientes portadores de hipertensão arterial secundária, de origem adrenal, por faixa etária (HUPE-UERJ).

\begin{tabular}{lcccccccc}
\hline & $\mathbf{0 - 9}$ & $\mathbf{1 0 - 1 9}$ & $\mathbf{2 0 - 2 9}$ & $\mathbf{3 0 - 3 9}$ & $\mathbf{4 0 - 4 9}$ & $\mathbf{5 0 - 5 9}$ & $\mathbf{6 0 - 6 9}$ & $>\mathbf{7 0}$ \\
\hline Feocromocitoma & 0 & 6 & 4 & 11 & 8 & 3 & 2 & 2 \\
Aldosteronoma & 0 & 1 & 1 & 2 & 6 & 5 & 1 & 0 \\
Síndrome de Cushing & 0 & 1 & 3 & 9 & 5 & 3 & 0 & 0 \\
\hline
\end{tabular}

Houve nítida predominância do sexo feminino (27 pacientes $-71 \%$ dos casos). A distribuição dos pacientes por faixa etária está ilustrada na Tabela 3 , mostrando preponderância de acometimento nas quarta e quinta décadas.

Em sua grande maioria, os pacientes apresentavam hipertensão arterial paroxística, associada a cefaléia e vômitos; um deles apresentava hipotensão postural, como aspecto clínico preponderante; naquele com feocromocitoma localizado em bexiga, a descarga de catecolaminas era evidenciada à micção. A quase totalidade dos pacientes foi submetida a bloqueio alfa-adrenérgico pré-operatório com fenoxibenzamina (20-40 mg/dia, em duas tomadas), seguido por bloqueio betaadrenérgico com propranolol (40-120 mg/dia, em duas tomadas). Benzodiazepínicos foram utilizados, na dose de $30 \mathrm{mg} /$ dia (em três tomadas), nos três dias que antecederam as operações. Todos foram submetidos à administração intravenosa adicional de fluidos, nos dois dias prévios às operações. $\mathrm{O}$ controle per-operatório da hipertensão desencadeada pela manipulação tumoral foi conseguido com gotejamento de fentolamina; a hipotensão pós-ligadura da veia adrenal direita, em alguns casos, suscitou a utilização de adrenalina intravenosa (aliado a reposição copiosa de fluidos intravenosos).

Trinta e cinco tumores eram benignos $(92,1 \%$ dos casos), e apenas três malignos: um paciente com metástases hepáticas à operação e dois outros com recorrência tumoral (metástases hepáticas), ambos reoperados cerca de um ano após a primeira intervenção cirúrgica.

Somente os três últimos pacientes de nossa casuística foram operados por via de acesso laparoscópica transabdominal (dois com tumores em adrenal direita e um à esquerda). Desses, os dois primeiros pacientes não apresentaram complicações; a última paciente, ainda na fase de recuperação intra-hospitalar, curiosamente, apresentou quadro de apendicite aguda, sendo realizado apendicectomia (também por vídeolaparoscópia), com boa evolução. Os demais foram submetidos a operações por via de acesso aberta transabdominal (incisões medianas supra- e/ou infra-umbilicais ou subcostais direita e/ou esquerda). Nesses últimos pacientes, algumas complicações ocorreram: duas nefrectomias (por infiltração renal, em casos de doenças malignas), uma esplenectomia (por laceração esplênica acidental de grande monta) e um óbito per-operatório (por sangramento incontrolável, durante adrenalectomia direita).

No período pós-operatório imediato, ainda na Unidade Intensiva, em todos os pacientes ocorreu a normalização da pressão arterial, com ausência de paroxismos. Nos dois pacientes em que houve a recorrência tumoral (por metástases hepáticas), a hipertensão arterial paroxística tornou a surgir nove e dez meses, após a operação inicial.

\section{ALDOSTERONISMO PRIMÁRIO - SÍNDROME DE CONN}

O adenoma adrenal é a principal causa desta enfermidade ( $82 \%$ a $92 \%)$, mas ela também pode se dever a neoplasia maligna e hiperplasia do córtex glandular. Com adenomas, a ressecção cirúrgica comumente leva à regressão da hipocalemia ( $88 \%$ a $100 \%$ dos casos $)^{7,10,38}$. embora hipertensão arterial persistente, mas de controle mais facilitado, possa ocorrer em $12 \%$ a $34 \%$ dos pacientes, mesmo na ausência de alteração hormonal recorrente ${ }^{39}$. A etiologia para essa hipertensão persistente ainda não foi determinada, mas acredita-se que o quadro clínico de longo tempo, a coexistência da hipertensão arterial primária, a idade e a história familiar de hipertensão podem determinar essa ocorrência ${ }^{39,40}$.

Em contraposição, quando a doença é causada por hiperplasia glandular (usualmente bilateral), a efetividade do tratamento cirúrgico (adrenalectomia subtotal) é baixa, reservando-se a indicação operatória somente para aqueles casos em que o tratamento medicamentoso com espironolactona ou outros fármacos não seja eficaz ${ }^{18,38}$. Somente em certos tipos de hiperplasia localizados em uma única glândula, nãoresponsivos à terapia medicamentosa, a ressecção cirúrgica pode, eventualmente, apresentar bons resultados ${ }^{18}$.

As vias de acesso são as mesmas já mencionadas anteriormente. Contudo, na atualidade, a ressecção total ou parcial de aldosteronomas constitui uma das principais indicações para a utilização da via de acesso laparoscópica (transabdominal ou retroperitoneal), com resultados comparáveis ao da técnica aberta, mas com recuperação pós-operatória muito mais precoce, tornando-a a principal escolha. Esse fato se deve a que, nesta enfermidade, os tumores são geralmente pequenos $(<3 \mathrm{~cm})$, sendo rara a ocorrência de tumores maiores que $6 \mathrm{~cm}^{7,10,12,18,39}$. Como a unilateralidade da lesão é a regra, no que se refere a aldosteronomas, a utilização da via de acesso posterior, com ressecção da 12. a costela (procedimento de Young), também encontra indicação, mas também vem sendo preterida, a favor da ressecção laparoscópica ${ }^{7,38}$.

$\mathrm{O}$ único fator nitidamente associado com uma resposta favorável após a adrenalectomia por aldosteronismo primário, é o achado histopatológico de um adenoma cortical. Embora alguns casos de hiperplasia também possam ser curados ou parcialmente controlados com a operação, possivelmente devido à redução de células da camada glandular 
glomerulosa, os resultados usualmente são bastante inferiores $^{18}$. Outros fatores associados com a cura são a resposta pré-operatória à espironolactona, a idade inferior a 44 anos, $\mathrm{o}$ tempo de duração da hipertensão (quanto menor, melhor a resposta) e a ausência de história familiar para hipertensão ${ }^{41}$.

Nos últimos 32 anos (1973-2005), 16 pacientes portadores de aldosteronismo primário foram operados no Hospital Universitário Pedro Ernesto (Departamento de Cirurgia Geral - Faculdade de Ciências Médicas - UERJ). Foram 12 adenomas, um carcinoma e três hiperplasias glandulares (esses três últimos operados no início da casuística). Os adenomas localizaram-se, preferencialmente, na glândula adrenal direita (oito pacientes - 66,6\%); o único carcinoma, estava localizado na glândula esquerda (idade - 45 anos). Ocorreu preponderância do sexo feminino, com 12 pacientes (75\%). A idade variou entre 17-69 anos, com prevalência nas quinta e sexta décadas (Tabela 3).

Os três pacientes com hiperplasia glandular apresentaram persistência da hipertensão arterial, mas com controle facilitado com o uso de anti-hipertensivos. Dos pacientes com adenomas, à exceção do paciente mais idoso (69 anos), todos apresentaram abolição do quadro clínico e laboratorial. Esse único paciente apresentava hipertensão arterial de longa data, no pré-operatório, e persistiu hipertenso (embora em menor grau), em fácil controle com anti-hipertensivos. Em outro desses pacientes com adenomas, como persistia hipertenso, após 30 dias de pós-operatório, detectou-se um tumor na glândula contralateral; foi reoperado, advindo abolição dos sinais e sintomas.

Somente os três últimos pacientes de nossa casuística foram operados por via de acesso laparoscópica transabdominal (dois com tumores em adrenal esquerda e um à direita). Os demais foram submetidos a operações por variadas vias de acesso aberto: nas hiperplasias glandulares, foram realizadas incisões medianas supra-umbilicais; no paciente com carcinoma, a incisão utilizada foi a subcostal; nos demais, incisão subcostal ipsilateral ou incisões posteriores, com ressecção da $12 .^{a}$ costela.

\section{SÍNDROME DE CUSHING}

A síndrome de Cushing é causada pela hipersecreção de adrenocorticotropina (ACTH) (síndrome de Cushing dependente de $\mathrm{ACTH}$ - doença de Cushing), pela hipersecreção adrenal primária de glicocorticóides (síndrome de Cushing independente de $\mathrm{ACTH}$ ). ou pela secreção ectópica de $\mathrm{ACTH}^{42}$. Inicialmente, a adrenalectomia era utilizada para tratamento de ambas as situações, síndrome e doença de Cushing, de origem adrenal ou hipofisária, respectivamente. Posteriormente, a ressecção transesfenoidal do adenoma pituitário mostrouse superior à adrenalectomia, para tratamento da doença de Cushing (dependente de ACTH) ${ }^{43}$.

Dos pacientes com síndrome de Cushing, 2/3 são causadas pela doença de Cushing, em que, na maioria dos casos (aproximadamente 90\%), microadenomas pituitários estão presentes ${ }^{44}$. A secreção ectópica de $\mathrm{ACTH}$, responsável por cerca de $15 \%$ dos casos de síndrome de Cushing, está freqüentemente associada com carcinomas de pulmão e ou- tras neoplasias, tumores carcinóides e outros tumores endócrinos (incluindo feocromocitoma) ${ }^{42}$.

$\mathrm{Na}$ atualidade, a adrenalectomia está indicada para pacientes com doença na glândula supra-renal (20\% a $30 \%$ dos casos), síndrome ectópica de secreção de ACTH ou na doença hipofisária refratária a outros tratamentos ${ }^{44}$. Nessas duas últimas situações, quando indicada, realiza-se a adrenalectomia total bilateral.

A síndrome de Cushing decorrente de doença na glândula supra-renal pode se dever a adenomas (9\% a $15 \%$ dos casos), carcinomas ( $5 \%$ a $10 \%$ dos casos) ou hiperplasia adrenal primária (aproximadamente $5 \%$ dos casos) ${ }^{42,44}$. Adenomas, geralmente, apresentam diâmetros menores do que $5 \mathrm{~cm}$; lesões maiores, freqüentemente, são malignas, na maioria das vezes levando ao surgimento de síndrome adrenogenital. Nesses casos, a adrenalectomia (total ou subtotal, uni- ou bilateral, conforme o acometimento) é a opção preferencial para o tratamento da síndrome de Cushing ${ }^{44}$.

Até cerca de 1970, a via de acesso de escolha era a transabdominal (por incisão mediana ou subcostal). A operação aberta, tanto na síndrome quanto na doença de Cushing, com utilização de grandes incisões, em pacientes com alteração da cicatrização e resistência reduzida à infecção, estava associada a mais de $40 \%$ de complicações (hérnias incisionais, esplenectomias incidentais, quadros infecciosos intra-abdominais e da ferida operatória, e hemorragia pós-operatória, dentre outras) e taxas de mortalidade entre $2 \%$ e $6 \%{ }^{45}$. Com o tempo, as vias de acessos posteriores e laterais (flancos), com abordagem retroperitoneal das lesões, passaram a ser as mais utilizadas. Essas incisões eram utilizadas mesmo quando a indicação era a adrenalectomia bilateral, necessitando mudança de posicionamento na mesa operatória para abordagem da glândula contralateral. Desde meados da década de 1990, contudo, a adrenalectomia laparoscópica retroperitoneal passou a ser o tratamento de escolha para as lesões benignas ${ }^{42,44}$.

Nos últimos 30 anos (1970-2000), 21 pacientes portadores de síndrome de Cushing decorrente de doença na glândula supra-renal foram operados no Hospital Universitário Pedro Ernesto (Departamento de Cirurgia Geral - Faculdade de Ciências Médicas - UERJ). Foram 12 adenomas, dois carcinomas e sete hiperplasias glandulares (esses sete últimos operados na primeira década da casuística). Os adenomas apresentaram distribuição quase equivalente, nas duas glândulas, com sete localizando-se à esquerda e seis à direita; os dois carcinomas estavam localizados na glândula direita (pacientes com 42 e 56 anos). Ocorreu preponderância do sexo feminino, com 17 pacientes (80,9\%). A idade variou entre 17-56 anos, com prevalência nas quarta e quinta décadas (Tabela 3 ).

Quatro dos pacientes com hiperplasia glandular, que persistiram hipertensos após a adrenalectomia, foram posteriormente operados para ressecção transesfenoidal de microadenomas hipofisários. Dos pacientes com adenomas, em oito, a pressão arterial retornou ao normal, além de regressão importante de sinais da síndrome de Cushing; um persistiu com hipertensão de fácil controle; os dois outros evoluíram para o óbito, um per-operatório (por sangramento intenso incontrolável) e outro no pós-operatório recente (com quadro de sepse abdominal por peritonite aguda grave). Os dois paci- 
entes com carcinoma adrenocortical apresentavam tumores de grande extensão, falecendo ainda dentro do primeiro ano de pós-operatório.

Nenhum dos pacientes de nossa casuística foi operado por vídeolaparoscópia (possivelmente porque, nos últimos cinco anos, nenhum paciente com síndrome de Cushing foi operado no nosso serviço). Todos foram submetidos a operações por variadas vias de acesso aberto: nas hiperplasias glandulares, foram realizadas incisões medianas supra-umbilicais; nos adenomas, a via de acesso variou entre a incisão mediana e as incisões posteriores, com ressecção da $12 .{ }^{a}$ costela; um dos pacientes com carcinoma foi operado por incisão mediana supra-umbilical, e o outro, que evoluiu para óbito durante o procedimento cirúrgico, por incisão posterior.

\section{CONSIDERAÇÕES FINAIS}

O tratamento cirúrgico das afecções da glândula supra-renal que determinam a ocorrência de hipertensão arterial secundária é, no mais das vezes, gratificante, propiciando a cura dos pacientes ou, ao menos, um controle mais facilitado da doença endócrina. A cuidadosa seleção de pacientes, o adequado preparo pré-operatório e a técnica operatória primorosa são essenciais para se alcançar essa meta. Há cerca de dez anos, a via de acesso para tratamento da maioria desses tumores vem sofrendo uma nítida mudança, notadamente daqueles com até $6 \mathrm{~cm}$ de diâmetro, passando da abordagem aberta (convencional) para a abordagem laparoscópica, com importantes benefícios para os pacientes.

\begin{abstract}
Among the main etiologies of secondary arterial hypertension figure out the tumorous affections of adrenal gland, located on cortex - primary aldosteronism (Conn's syndrome) and Cushing's syndrome - or at glandular medulla - pheocromocytoma. Although these tumors are at most benign the surgical resection is needed in order to eliminate the disturbances provided by them and to limit the mass growth, being curative in about 80-90\% of the cases. In this paper some particularities above surgical treatment of these diseases will be focused emphasizing the pre-operative prepare of the patients and the currently preconized approach (Rev. Col. Bras. Cir. 2006; 33(6): 401-410).
\end{abstract}

Key-words: Hypertension; Pheocromocytoma; Adrenal glands; Cushing Syndrome; Adenoma; Surgery.

\section{REFERÊNCIAS}

1. Pacak K, Linehan WM, Eisenhofer G, Walther MM, Goldstein DS. Recent advances in genetics, diagnosis, localization, and treatment of pheochromocytoma. Ann Intern Med. 2001;134(4):315-29.

2. Plouin PF, Duclos JM, Soppelsa F, Boublil G, Cahtellier G. Factors associated with perioperative morbidity and mortality in patients with pheochromocytoma: analysis of 165 operations at a single center. J Clin Endocrinol Metab. 2001; 86(4):1480-6.

3. Bentrem DJ, Pappas SG, Ahuja Y, Murayama KM, Angelos P. Contemporary surgical management of pheochromocytoma. Am J Surg. 2002;184(6):621-4; discussion 624-5.

4. Gagner M, Lacroix A, Bolte E. Laparoscopic adrenalectomy in Cushing's syndrome and pheochromocytoma. N Engl J Med. 1992; 327(14):1033.

5. Prinz RA. A comparison of laparoscopic and open adrenalectomies. Arch Surg. 1995;130(5):489-92; discussion 4924.

6. Dudley NE, Harrison BJ. Comparison of open posterior versus transperitoneal laparoscopic adrenalectomy. Br J Surg. 1999;86(5):656-60.

7. Assalia A, Gagner M. Laparoscopic adrenalectomy. Br J Surg. 2004;91(10):1259-74.

8. Schell SR, Talamini MA, Udelsman R. Laparoscopic adrenalectomy for nonmalignant disease: improved safety, morbidity, and cost-effectiveness. Surg Endosc. 1999;13(1):30-4.

9. Gagner M, Pomp A, Heniford BT, Pharand D, Lacroix A. Laparoscopic adrenalectomy: lessons learned from 100 consecutive procedures. Ann Surg. 1997;226(3):238-46; discussion 246-7.
10. Lezoche E, Guerrieri M, Paganini AM, Feliciotti F, Zenobi P, Antognini F, Mantero F. Laparoscopic adrenalectomy by the anterior transperitoneal approach: results of 108 operations in unselected cases. Surg Endosc. 2000;14(10):920-5.

11. MacGillivray DC, Whalen GF, Malchoff CD, Oppenheim DS, Shichman SJ. Laparoscopic resection of large adrenal tumors. Ann Surg Oncol. 2002;9(5):480-5.

12. Miccoli P, Raffaelli M, Berti P, Materazzi G, Massi M, Bernini G. Adrenal surgery before and after the introduction of laparoscopic adrenalectomy. Br J Surg. 2002;89(6):779-82.

13. Gill IS, Hobart MG, Schweizer D, et al. Outpatient adrenalectomy. J Urol. 2000; 163(3):717-20.

14. Edwin B, Raeder I, Trondsen E, Kaaresen R, Buanes T. Outpatient laparoscopic adrenalectomy in patients with Conn's syndrome. Surg Endosc. 2001;15(6):589-91. Epub 2001 Mar 13.

15. Gill IS, Sung GT, Hsu TH, Meraney AM. Robotic remote laparoscopic nephrectomy and adrenalectomy: the initial experience. J Urol. 2000;164(6):2082-5.

16. Young JA, Chapman WH $3^{\text {rd }}$, Kim VB, Albrecht RJ, Nq PC, Nifong LW, Chitwood WR Jr. Robotic-assisted adrenalectomy for adrenal incidentaloma: case and review of the technique. Surg Laparosc Endosc Percutan Tech. 2002;12(2):126-30.

17. Ortiz V, Kiehl R. Glândulas supra-renais. In: Petroianu A, editor. Anatomia cirúrgica. 1. ${ }^{\text {a }}$ ed. Rio de Janeiro: Guanabara Koogan; 1999. p. 546-8.

18. Desai MM, Gill IS. Primary aldosteronism - Surgical approaches. In: Mansoor GA, editor. $1^{\text {st }}$ ed. Secondary hypertension. New Jersey: Human Press; 2004. p. 149-76.

19. Kinney MA, Narr BJ, Warner MA. Perioperative management of pheochromocytoma. J Cardiothorac Vasc Anesth. 2002;16(3):359-69. 
20. Goldstein RE, O’Neill JA Jr, Holcomb GW $3^{\text {rd }}$, Morgan WM $3^{\text {rd }}$, Neblett WW $3^{\text {rd }}$, Oates JA, Brown N, Nadeau J, Smith B, Page DL, Abumrad NN, Scott HW Jr. Clinical experience over 48 years with pheochromocytoma. Ann Surg. 1999;229(6):755-64; discussion 764-6.

21. Suzuki K. Surgical management of pheochromocytoma. Biomed Pharmacother. 2000; 54 (Suppl 1):150S-6S.

22. Connery LE, Coursin DB. Assessment and therapy of selected endocrine disorders. Anesthesiol Clin North Am. 2004; 22(1):93-123.

23. Bravo EL. Pheochromocytoma. Curr Ther Endocrinol Metab. 1997;6:195-7.

24. Welbourn RB. Early surgical history of phaeochromocytoma. $\mathrm{Br}$ J Surg. 1987;74(7):594-6.

25. Malchoff CD, MacGillivray D, Shichman S. Pheochromocytoma treatment. In: Mansoor GA, editor. $1^{\text {st }}$ ed. Secondary hypertension. New Jersey: Human Press; 2004. p. 235-49.

26. Sprung J, O'Hara JF Jr, Gill IS, Abdelmalak B, Sarnaik A, Bravo EL. Anesthetic aspects of laparoscopic and open adrenalectomy for pheochromocytoma. Urology. 2000; 55(3):339-43.

27. Fernandez-Cruz L, Taura P, Saenz A, Benarroch G, Sabater L. Laparoscopic approach for pheochromocytoma: hemodynamic changes and catecholamine secretion. World J Surg. 1996;20(7):762-8; discussion 768.

28. Orchard T, Grant CS, van Heerden JA, Weaver A, Irvin GL, Pommier R, Doppman JL. Pheochromocytoma: continuing evolution of surgical therapy. Surgery. 1993;114(6):1153-9.

29. Kinney MA, Warner MA, van Heerden JA, Horlocker TT, Young Jr WF, Schroeder DR, Maxson PM, Warner MA. Perianesthetic risks and outcomes of pheochromocytoma and paraganglioma resection. Anesth Analg. 2000;91(5):1118-23.

30. Prys-Roberts C. Phaeochromocytoma - recent progress in its management. Br J Anaesth. 2000; 85(1):44-57. Erratum in: Br J Anaesth 2001;86(4):605.

31. Pullerits J, Ein S, Balfe JW. Anesthesia for phaeochromocytoma. Can J Anaesth. 1988; 35(5):526-34.

32. Shupak RC. Difficult anesthetic management during pheochromocytoma surgery. J Clin Anesth .1999;11(3):247-50.

33. Sloand EM, Thompson BT. Propranolol-induced pulmonary edema and shock in a patient with pheochromocytoma. Arch Intern Med. 1984;144(1):173-4.

34. Steinsapir J, Car AA, Prisant LM, Bransome ED Jr. Metyrosine and pheochromocytoma. Arch Intern Med. 1997;157(8):901-6.

35. Roizen MF, Hunt TK, Beaupre PN, Kremer P, Firmin R, Chang CN, Alpert RA, Thomas CJ, Tyrrell JB, Cahalan MK The effect of alpha-adrenergic blockade on cardiac performance and tissue oxygen delivery during excision of pheochromocytoma. Surgery. 1983;94(6):941-5.
36. Boutros AR, Bravo EL, Zanettin G, Straffon RA. Perioperative management of 63 patients with pheochromocytoma. Cleve Clin J Med. 1990; 57(7):613-7.

37. Pivalizza EG. Magnesium sulfate and epidural anesthesia in pheochromocytoma and severe coronary artery disease. Anesth Analg. 1995;81(2):414-6.

38. Miyake O, Okuyama A. Surgical management of primary aldosteronism. Biomed Pharmacother. 2000; 54(Suppl 1):S1469.

39. Rossi H, Kim A, Prinz RA. Primary hyperaldosteronism in the era of laparoscopic adrenalectomy. Am Surg. 2002;68(3):253-6; discussion 256-7.

40. Young WF Jr. Primary aldosteronism: management issues. Ann N Y Acad Sci. 2002; 970:61-76.

41. Sawka AM, Young WF Jr, Thompson GB, Grant CS, Farley DR, Leibson C, van Heerden JA. Primary aldosteronism: factors associated with normalization of blood pressure after surgery. Arch Intern Med. 2001;135(4):258-61.

42. Whithworth JA, Mangos GJ, Kelly JJ. Hypertension in Cushing's syndrome. In: Mansoor GA, editor. Secondary hypertension. $1^{\text {st }}$ ed. New Jersey: Human Press; 2004. p. 195-220.

43. Hardy J. Transphenoidal microsurgery of the normal and pathological pituitary. Clin Neurosurg. 1969;16:185-217.

44. Norton JA, Li M, Gillary J, Le HN. Cushing's syndrome. Curr Probl Surg. 2001;38(7):487-545.

45. Chapuis Y, Pitre J, Conti F, Abboud B, Pras-Jude N, Luton JP. Role and operative risk of bilateral adrenalectomy in hypercortisolism. World J Surg. 1996;20(7):775-9; discussion 779-80.

Como citar este artigo:

Marques RG, Sanjuliani AF. Tratamento cirúrgico da hipertensão arterial secundária com origem na glândula supra-renal. Rev Col Bras Cir. [periódico na Internet]. 2006 Nov-Dez;33(6). Disponível em URL: www.scielo.br/rcbc

Endereço para correspondência:

Ruy Garcia Marques

Rua Dr. Crespo 10

Condomínio Porto Fino 20, casa 01

Recreio

22790-670- Rio de Janeiro - RJ

E-mail: Rmarques@uerj.br 